\title{
Magnetism in $\mathrm{CeFeAsO}_{1-x} \mathrm{~F}_{x}$ and $\mathrm{LaFeAsO}_{1-x} \mathrm{~F}_{x}$ from first principles
}

\author{
S. Sharma, ${ }^{1,2,3, *}$ S. Shallcross, ${ }^{4}$ J. K. Dewhurst, ${ }^{1,2,3}$ A. Sanna, ${ }^{2,5}$ C. Bersier, ${ }^{1,2,3}$ S. Massidda, ${ }^{5}$ and E. K. U. Gross ${ }^{2,3}$ \\ ${ }^{1}$ Fritz Haber Institute of the Max Planck Society, Faradayweg 4-6, D-14195 Berlin, Germany \\ ${ }^{2}$ Institut für Theoretische Physik, Freie Universität Berlin, Arnimallee 14, D-14195 Berlin, Germany \\ ${ }^{3}$ European Theoretical Spectroscopy Facility (ETSF) \\ ${ }^{4}$ Lehrstuhl für Theoretische Festkörperphysik, Staudstr. 7-B2, 91058 Erlangen, Germany \\ ${ }^{5}$ SLACS-INFM/CNR and Dipartimento di Scienze Fisiche, Universita' degli Studi di Cagliari, I-09042 Monserrato (CA), Italy
}

(Received 18 August 2009; revised manuscript received 6 October 2009; published 4 November 2009)

\begin{abstract}
Using state-of-the-art first-principles calculations we study the magnetic behavior of CeOFeAs. We find the Ce layer moments oriented perpendicular to those of the Fe layers. An analysis of incommensurate magnetic structures reveals that the Ce-Ce magnetic coupling is rather weak with, however, a strong $\mathrm{Fe}-\mathrm{Fe}$ and $\mathrm{Fe}-\mathrm{Ce}$ coupling. Comparison of the origin of the tetragonal to orthorhombic structural distortion in $\mathrm{CeOFeAs}$ and LaOFeAs shows marked differences; in CeOFeAs the distortion is stabilized by a lowering of spectral weight at the Fermi level, while in LaOFeAs by increase in Fe spin moment. Finally, we investigate the impact of electron doping upon $\mathrm{CeOFeAs}$ and $\mathrm{LaOFeAs}$ and show that (a) while in $\mathrm{CeOFeAs}$ the ground-state $\mathrm{Fe}$ moment remains largely unchanged by doping, the stability of magnetic order goes to zero at a doping that corresponds well to the vanishing of the Néel temperature and, (b) in contrast the LaOFeAs system remains magnetic with a slowly vanishing moment as a function of doping.
\end{abstract}

DOI: 10.1103/PhysRevB.80.184502

PACS number(s): 74.25.Jb, 67.30.hj, 75.30.Fv

\section{INTRODUCTION}

The recently discovered ${ }^{1,2}$ family of FeAs-based compounds that, upon electron doping, become superconducting with transition temperatures up to $55 \mathrm{~K}$, are attracting a lot of interest. Structurally these materials $R \mathrm{OFeAs}(R=\mathrm{La}, \mathrm{Ce}, \mathrm{Pr}$, $\mathrm{Nd}$, and $\mathrm{Sm}$ ) are very similar in that they are formed from FeAs layers separated by rare earth or lanthanide oxide layers. In striking contrast to the well-known cuprates, these materials are magnetic metals with small itinerant moment, leading to the possibility of large spin-fluctuation effects. ${ }^{3,4}$ In particular, at the onset of superconductivity the moment on the $\mathrm{Fe}$ atoms vanishes, and a key question concerns the possible role of such spin fluctuations in the superconducting transition. ${ }^{3,4}$

Despite the diverse set of rare-earth and lanthanide atoms involved in these materials, physically they share many similarities. In particular, (i) at temperatures around $\sim 150 \mathrm{~K}$ a structural phase transition from tetragonal to orthorhombic crystal symmetry occurs, (ii) this is then closely followed by a magnetic phase transition to a spin order antiferromagnetic (AFM) in nature, (iii) upon doping with florine the AFM order is suppressed and superconductivity appears. Furthermore, calculations reveal that (iv) the nonmagnetic Fermi surfaces are all strongly nested, ${ }^{3,5,6}$ and (v) that the moment of the $\mathrm{Fe}$ atoms depends critically upon the separation of the Fe layer from the adjacent As layer. $5,7,8$

Given the diversity of constituents involved in this class of materials, such uniformity of behavior appears, at first sight, somewhat surprising. The question then arises if the underlying physical mechanisms behind such phenomenon as the structural distortion or doping behavior are also the same. In the present work we investigate this by analyzing the properties of $\mathrm{CeOFeAs}$ as compared to the well-studied LaOFeAs. The choice of these two materials is motivated by the fact that among the rare-earth and lanthanide oxypnic- tides they show relatively large differences for two key physical properties; (i) the low-temperature moment of the $\mathrm{Fe}$ atom, $0.35 \mu_{B}$ in $\mathrm{LaOFeAs}$ is the lowest while in $\mathrm{CeOFeAs}\left(0.94 \mu_{B}\right)$ is the highest among all oxypnictides ${ }^{9-12}$ and (ii) the superconducting transition temperature also differs strongly; $26 \mathrm{~K}$ in LaOFeAs (Refs. 1 and 13) and $45 \mathrm{~K}$ in CeOFeAs. ${ }^{14-16}$

Remarkably, we find that the mechanism behind the structural phase transition in $\mathrm{CeOFeAs}$ and $\mathrm{LaOFeAs}$ is quite different; the former case being driven, essentially, by the one-electron energies, while the latter is driven by gain in the magnetization energy. ${ }^{8,17,18}$ In addition, the behavior upon electron doping is substantially different. We find that for $\mathrm{CeO}_{1-x} \mathrm{~F}_{x} \mathrm{Fe}$ As the moment is almost unchanged upon electron doping (a reduction of 3\% for $x=0.10$ ), whereas calculations of $\mathrm{LaO}_{1-x} \mathrm{~F}_{x} \mathrm{Fe}$ As have revealed a strong suppression of the moment $(90 \%$ at $x=0.10)$. This may be reconciled with the vanishing of the Néel temperature at $x=0.06$ by the fact that the magnetic order becomes metastable near this doping.

\section{METHODOLOGY}

In the present work all calculations are performed using the state-of-the-art full-potential linearized augmented plane wave (FPLAPW) method, ${ }^{19}$ implemented within the ELK code. ${ }^{20}$ To obtain the Pauli spinor states, the Hamiltonian containing only the scalar potential is diagonalized in the LAPW basis: this is the first-variational step. The scalar states thus obtained are then used as a basis to set up a second-variational Hamiltonian with spinor degrees of freedom. ${ }^{19}$ This is more efficient than simply using spinor LAPW functions, but care must be taken to ensure that there is a sufficient number of first-variational eigenstates for convergence of the second-variational problem. We use a shifted $\mathbf{k}$ mesh of $10 \times 10 \times 6$ and 260 states per $\mathbf{k}$ point which 


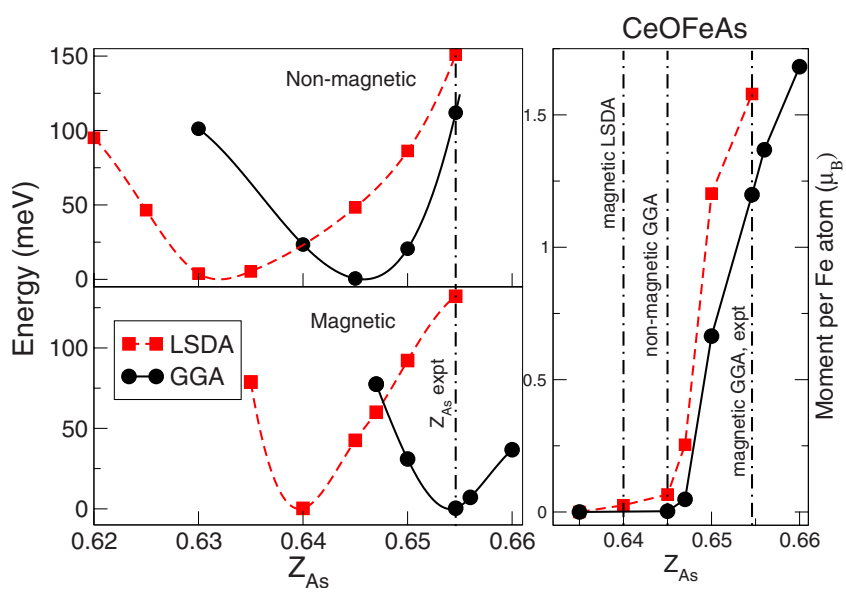

FIG. 1. (Color online) Top-left panel: energy (in meV) per formula unit as a function of position of the As atom calculated using LDA and GGA. Bottom-left panel: same as upper panel but the calculation is spin polarized. Right-hand panel shows the moment per Fe atom (in $\mu_{B}$ ) calculated using LSDA and GGA functionals.

ensures convergence of the second-variational step as well as the convergence with respect to the $\mathbf{k}$ points. All the experimental lattice parameters for CeOFeAs are from Ref. 14 and for $\mathrm{LaOFeAs}$ from Ref. 21

\section{GROUND STATE FOR UNDOPED UNDISTORTED CeOFeAs}

We first consider the undistorted undoped ground state of $\mathrm{CeOFeAs}$. One of the most striking aspects of the theoretically intensively studied compound LaOFeAs has been the spread of results for magnetic properties, attributed to both an unusual sensitivity to the approximation to exchange correlation, and a sensitive dependence upon the separation of the $\mathrm{Fe}$ and As layers, $z_{\mathrm{As}}{ }^{5,7,8}$ This latter behavior is also found in the case of $\mathrm{CeOFeAs},{ }^{22}$ however, in contrast to LaOFeAs a spin-polarized generalized gradient approximation (GGA) (Ref. 23) calculation yields an optimized $z_{\mathrm{As}}$ in near perfect agreement with experiment, see Fig. 1. Given this choice of $z_{\mathrm{As}}$ we now determine the ground-state magnetic structure for undoped undistorted $\mathrm{CeOFeAs}$. In order to study the possible incommensurate spin structures we have calculated the total energy as a function of the spin-spiral vector $\mathbf{q}$, for various directions in the Brillouin zone (BZ), using the local-spin-density approximation (LSDA). ${ }^{24}$ For the in-plane spin spirals, going in the direction $[0.5,0.5,0]$ to $[1,1,0]$, we find a clear sharp minimum at the commensurate $\mathbf{q}$ vector of $[0.5,0.5,0]$, equivalent to stripe AFM spin configuration (top panel Fig. 2). From Fig. 2 it is also clear that the various spin configurations are almost degenerate in the direction $[0.5,0.5,0.4]$ to $[0.5,0.5,0.5]$. This is indicative of weak interplane coupling [coupling between one $\mathrm{Fe}(\mathrm{Ce})$ layer with the next $\mathrm{Fe}(\mathrm{Ce})$ layer]; this finding is concomitant with experimental results of Zhao et al. ${ }^{14}$ This is a good news as far as the computational effort is concerned since this would mean that it is not essential to double the unit cell in $c$ axis for collinear calculations. Experimentally ${ }^{14}$ it is known that below a temperature of $4 \mathrm{~K}$ the Ce moments

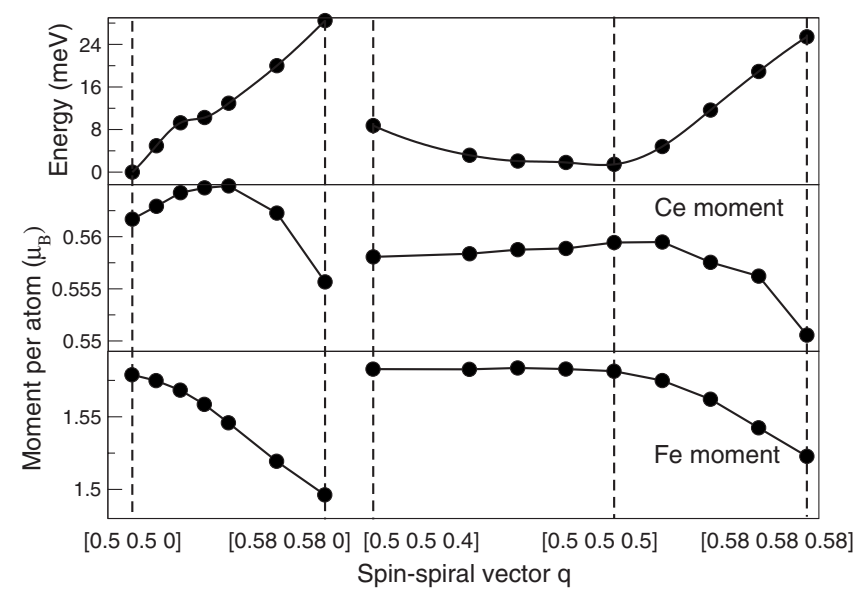

FIG. 2. Top panel shows the energy (in meV per formula unit) and the middle and bottom panels show the magnetic moment (in $\mu_{B}$ ) per $\mathrm{Ce}$ and $\mathrm{Fe}$ atom, respectively. All quantities are plotted as a function of the spin-spiral $q$ vector.

couple strongly to the $\mathrm{Fe}$ moments, interestingly, we also find this to be the case (all present calculations are performed at $0 \mathrm{~K})$. Indications of this reasonably strong magnetic coupling between $\mathrm{Fe}$ and $\mathrm{Ce}$ atoms are; (a) a small change (1\%) of the Ce moment (performed with a fixed spin calculation), leads to a large change $(3 \%)$ in the Fe moment and (b) to change Fe moment by $1.3 \%$ (Ce moment kept fixed to zero) requires $7 \mathrm{meV}$ energy, while similar change in Fe moment with $\mathrm{Ce}$ atoms treated magnetically requires larger $(11 \mathrm{meV})$ energy. This extra cost in energy in the later case is due to the localized nature of the $\mathrm{Ce}$ moments which via magnetic coupling tries to preserve the moment on the Fe atoms. This mechanism is local in that we find similar behavior for several different long-range orders of the Ce sublattice (ferromagnetic, as well as several different spin-spiral vectors). Such a weak dependence on the nature of the long-range order suggests that also for disordered spin arrangements similar behavior will be seen, an important point since at all temperatures of interest the Ce sublattice will have substantial spin disorder.

In addition to the LSDA functional, the use of GGA and LSDA $+U$ functionals (with $U=6 \mathrm{eV}$ and $J=1 \mathrm{eV}$ for the Ce atom), also leads to the Fe layer adopting the stripe AFM structure found in all the oxypnictides, with the magnetic moments of the Ce layer perpendicular to those of the $\mathrm{Fe}$ layer. The $\mathrm{Fe}(\mathrm{Ce})$ moments are found to be $1.58 \mu_{B}$ $\left(0.56 \mu_{B}\right), 1.52 \mu_{B}\left(0.92 \mu_{B}\right)$, and $1.30 \mu_{B}\left(0.54 \mu_{B}\right)$ for the LSDA, LSDA $+U$, and GGA functionals, respectively, with the corresponding experimental values $0.94 \mu_{B}$ for $\mathrm{Fe}$ and $0.83 \mu_{B}$ for $\mathrm{Ce}$. The agreement with experiment for the $\mathrm{Fe}$ moment is thus rather poor for all functionals considered. This may be attributed to the exceptionally sensitive dependence of the $\mathrm{Fe}$ moment on $z_{\mathrm{As}}$, see Fig. 1, which for $\mathrm{CeOFeAs}$ is even more pronounced than in LaOFeAs. It is also worth mentioning that the moments listed above are spin moments and the orbital contributions, which require the presence of spin-orbit coupling in the Hamiltonian, are not added. Since in the present work main focus is on the possible incommensurate nature of spins all the calculations are 
performed using fully noncollinear spin-spiral formalism which can only be treated in absence of spin-orbit coupling.

Turning now to the spin-spiral moments (lower two panels Fig. 2), we find that the moment remains almost unchanged upon changing the magnetic structure away from the stripe AFM spin configuration. This is in striking contrast to $\mathrm{LaOFeAs}$, which remains spin polarized ${ }^{8,25,26}$ only in a small region about the $M$ point, $[0.5,0.5,0]$, with the moment vanishing elsewhere in the BZ. Since for $\mathrm{CeOFeAs}$ the moment on the $\mathrm{Fe}$ atoms changes only slightly- $5.2 \%$ upon moving across the BZ hence the role of spin fluctuations could be rather different in these two materials. This difference may be understood as a consequence of the nature of the various interplane and intraplane magnetic couplings in $\mathrm{CeOFeAs}$. In particular, the intraplane coupling of the $\mathrm{Ce}$ atoms is very weak-we find the energy difference between FM- and AFM-ordered in-plane Ce moments to be almost degenerate with, additionally, the magnitude of the Ce moments unchanged by the choice of FM or AFM order. Thus the nature of the Ce-Ce interaction results in the Ce moment remaining unchanged by a spin-wave configuration; this in turn acts to preserve the Fe moment due to the relatively strong interplane $\mathrm{Fe}-\mathrm{Ce}$ coupling. This weak $\mathrm{Ce}-\mathrm{Ce}$ coupling has yet another consequence; in order to study this system using a collinear formalism one does not need to construct a supercell in order to account for, the experimentally observed, different spin-spiral $\mathbf{q}$ vectors for the $\mathrm{Ce}$ and the $\mathrm{Fe}$ layers. At this point it is important to mention that these conclusions remain unchanged for all three exchangecorrelation functionals (LSDA, GGA, and $\mathrm{LSDA}+U$ ) used in the present work; despite the functional the ground state is AFM stripelike and among the magnetic atoms the strongest coupling is between intraplane $\mathrm{Fe}-\mathrm{Fe}$ atoms followed by interplane $\mathrm{Fe}-\mathrm{Ce}$ atoms. The intraplane $\mathrm{Ce}-\mathrm{Ce}$ and indirect interplane coupling between $\mathrm{Fe}-\mathrm{Fe}$ and $\mathrm{Ce}-\mathrm{Ce}$ atoms always remain very weak.

\section{STRUCTURAL DISTORTION IN CeOFeAs VS LaOFeAs}

As with all the oxypnictides, $\mathrm{CeOFeAs} \mathrm{undergoes} \mathrm{a} \mathrm{struc-}$ tural phase transition from tetragonal to orthorhombic crystal symmetry, ${ }^{14}$ which in this compound occurs at $160 \mathrm{~K}$. In order to determine the physical reason behind this transition we have performed $a b$ intio LSDA calculations. As may be seen in Fig. 3 the nonmagnetic compound does not show any crystal distortion, but upon performing spin-polarized calculations one finds a minimum at a distortion angle of $90.30^{\circ}$ (for definition of distortion angle see Refs. 8 and 17). This clearly shows that the magnetic interactions between Fe atoms lead to structural distortion. This is hardy surprising; the ground states for the undistorted CeOFeAs (AFM stripelike) is essentially magnetically frustrated, and one means of removal of the magnetic frustration is the structural distortion.

Thus both the distortion angle and critical temperature for $\mathrm{CeOFeAs}$ are very similar to those found in $\mathrm{LaOFeAs}$, however, as we will now show, the underlying reason for the lowering in energy due to distortion is entirely different in the two cases. In the case of $\mathrm{LaOFeAs}$ the crystal distortion leads to removal of magnetic frustration ${ }^{17,18}$ which in turn

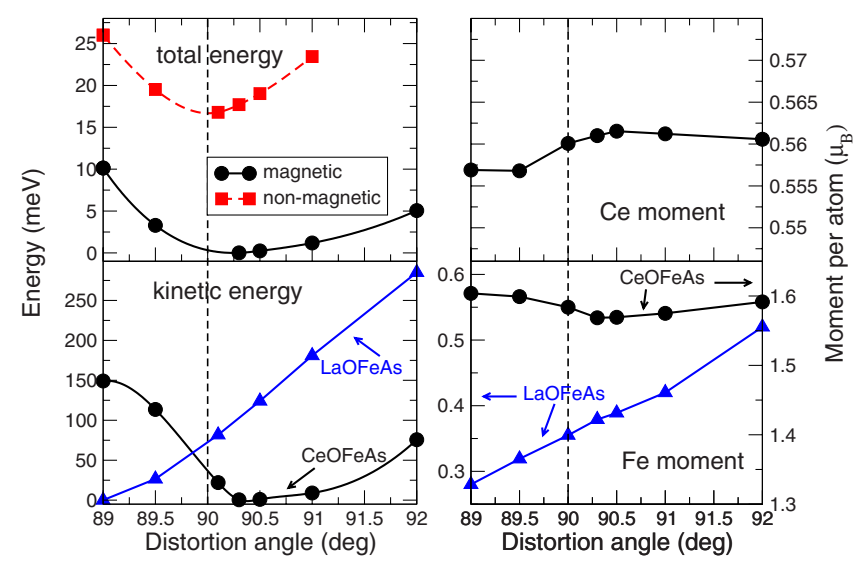

FIG. 3. (Color online) Top-left panel shows the total energy and bottom-left panel the one-electron kinetic energy (in meV) per formula unit. The right panel shows the moment per $\mathrm{Ce}$ (top) and $\mathrm{Fe}$ (bottom) atom (in $\mu_{B}$ ) as a function of distortion angle (in degrees).

leads to an increase in moment on the Fe atoms. This increase in moment causes a gain in magnetization energy which is then sufficient to make the distorted structure energetically preferred.$^{8}$ On the other hand, the moment of both the $\mathrm{Fe}$ and $\mathrm{Ce}$ atoms remains essentially unchanged in $\mathrm{CeOFeAs}$ upon distortion, see right-hand panels of Fig. 3. The reason for this lies again in the magnetic coupling between the $\mathrm{Fe}$ and $\mathrm{Ce}$ atoms; the moment on the $\mathrm{Ce}$ atom does not depend strongly upon the distortion angle due to weak intraplane $\mathrm{Ce}-\mathrm{Ce}$ coupling, this in turn preserves moment on the $\mathrm{Fe}$ atoms. The consequence of this is that the gain in magnetization energy is not sufficient to make the distorted structure favorable. However, a careful analysis of various contributions to the total energy (one-electron, electrostatic, exchange correlation, and magnetization) exhibits that all but the one-electron kinetic energy show a monotonic behavior as a function of the distortion angle. The one-electron energy of $\mathrm{CeOFeAs}$ shows a clear minimum at $90.30^{\circ}$ while (lowerleft panel Fig. 3), in dramatic contrast, the $\mathrm{LaOFeAs}$ oneelectron kinetic energy monotonically increases as a function of distortion angle. This points to the one-electron energies playing a leading role in the structural distortion of CeOFeAs. Recently, a similar mechanism was found in the case of $\mathrm{BaFe}_{2} \mathrm{As}_{2}$, where it was shown that the relevant physics with respect to structural distortion in oxypinctides lies in the one-electron energies. ${ }^{27}$

Since this striking contrast between $\mathrm{LaOFeAs}$ and $\mathrm{CeOFeAs}$ is linked to the one-electron energies this difference should also be apparent in the electronic band structure of the two materials. In this regard in Fig. 4 are shown the nonmagnetic as well as magnetic (AFM stripe phase) density of states (DOS) for the undistorted and distorted lattices of $\mathrm{CeOFeAs}$ and $\mathrm{LaOFeAs}$. One feature common to both compounds is that, as one would expect, spin polarization results in a large shift of spectral weight away from the Fermi level. Turning to the impact of distortion one observes a striking difference: in $\mathrm{LaOFeAs}$ there is a substantial increase in spectral weight at the Fermi level. This increase in the spectral weight upon distortion has been noticed before in Refs. 17 and 28. In contrast to this, in $\mathrm{CeOFeAs}$ spectral weight 


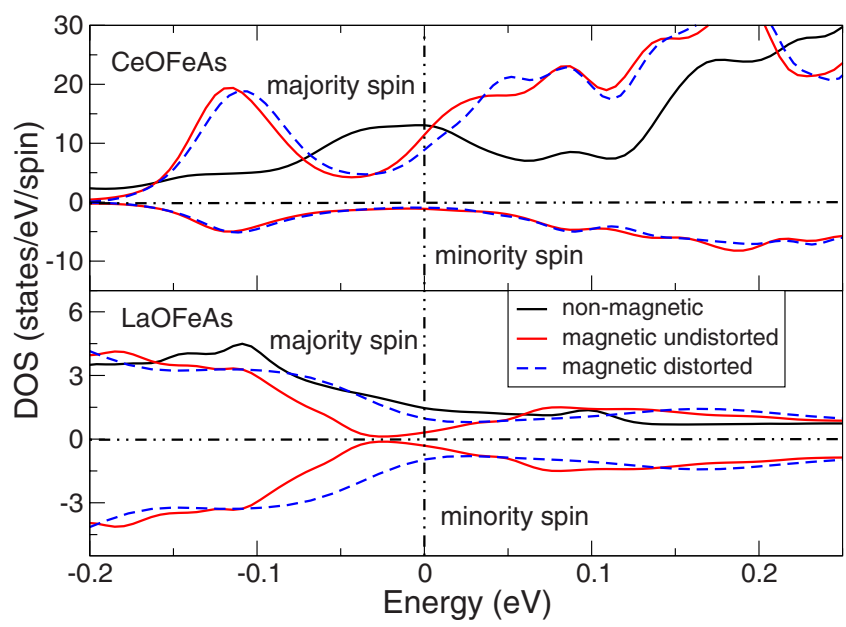

FIG. 4. (Color online) Density of states calculated using the LSDA for undistorted nonmagnetic, undistorted magnetic, and distorted magnetic structures for $\mathrm{CeOFeAs}$ and $\mathrm{LaOFeAs}$. Stripe antiferromagnetic order was used for the magnetic calculations.

moves away from the Fermi level. This scenario is similar to the one observed in the case of $\mathrm{BaFe}_{2} \mathrm{As}_{2} .{ }^{27}$ Clearly, therefore, although both the distortion angle and transition temperatures are very similar in these two oxypnictides, the origin of the structural phase transitions is very different. At this point it is also worth mentioning that heat-capacity measurements of McGuire et al. ${ }^{29}$ showed that this decrease in the DOS at the Fermi level in CeOFeAs on distortion is consistent with the Seebeck coefficient measurements.

\section{MAGNETISM IN ELECTRON-DOPED LaOFeAs VS CeOFeAs}

The most important property of the iron oxypnictides is the occurrence of a superconducting phase transition at a critical electron doping. ${ }^{14,15,30,31}$ An interesting difference between the temperature-doping phase diagrams of $\mathrm{CeO}_{1-x} \mathrm{~F}_{x} \mathrm{FeAs}$ and $\mathrm{LaO}_{1-x} \mathrm{~F}_{x} \mathrm{FeAs}$ is that, for the former case, the Néel temperature of the magnetic phase goes continuously to zero ${ }^{14}$ as critical doping is approached $\left(x_{c}\right.$ $=0.06$ ), while in $\mathrm{LaO}_{1-x} \mathrm{~F}_{x} \mathrm{FeAs}$ one instead finds a sharp drop $^{12,21,32,33}$ in the Néel temperature at a critical doping of $x_{c}=0.045$ (see right-hand panel Fig. 6). Concomitantly, lowtemperature measurements of the magnetic order of the $\mathrm{Fe}$ layer in $\mathrm{CeO}_{1-x} \mathrm{~F}_{x} \mathrm{FeAs}$ show that it is entirely lost before $x_{c}$ (left panel Fig. 6). The situation is slightly different for $\mathrm{LaO}_{1-x} \mathrm{~F}_{x} \mathrm{FeAs}$ in that, experimentally it is established that beyond $x=0.075$ no magnetic order persists, however, if magnetic order is entirely lost before the superconducting transition is still a point of discussion. ${ }^{12,21,32}$ An important question for understanding the role of magnetism in the superconducting transition is then whether it is the moment of the $\mathrm{Fe}$ atoms that vanishes, or whether it is simply the stripe AFM order that vanishes.

To investigate this issue we calculate $\mathrm{CeO}_{1-x} \mathrm{~F}_{x} \mathrm{FeAs}$ and $\mathrm{LaO}_{1-x} \mathrm{~F}_{x} \mathrm{Fe}$ As by deploying the virtual crystal approximation (VCA). Although the VCA neglects much of the physics of disorder, it has been shown recently ${ }^{34}$ to provide a surpris-

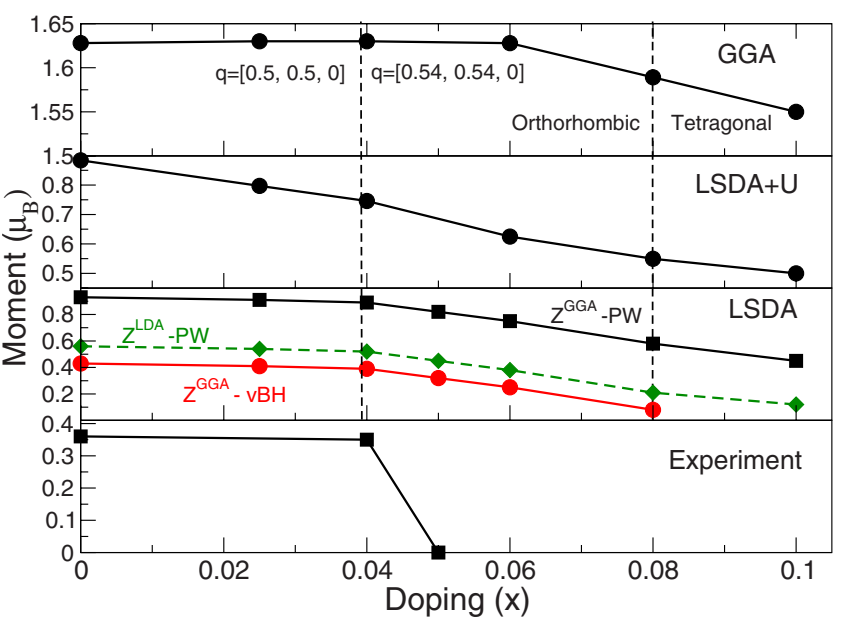

FIG. 5. (Color online) Moment per Fe atom (in $\mu_{B}$ ) as a function of doping calculated using two different parameterizations of LSDA, LSDA $+U$, and GGA functionals. The experimental data are taken from Ref. 21

ingly accurate account of magnetism and Fermiology in $\mathrm{LaO}_{1-x} \mathrm{~F}_{x} \mathrm{FeAs}$. In order to determine the impact of the approximation to the $x c$ functional, calculations are performed using the LSDA, LSDA $+U$, and GGA functionals.

We first consider the overall behavior of the magnetic moment with doping for $\mathrm{LaO}_{1-x} \mathrm{~F}_{x} \mathrm{FeAs}$. This is shown in Fig. 5 for GGA and LSDA $+U$, and two different parameterizations, von-Barth-Hedin (vBH) (Ref. 35) and Perdew-Wang, ${ }^{24}$ of the LSDA. It is immediately apparent that the choice of functional and $z_{\mathrm{As}}$ simply leads to a scaling of the doping curve but the over all behavior remains the same; like in experiments, the change in moment is very small for $x<0.05$, i.e., less than the critical doping. However, a marked divergence between experiment and theory occurs after this point; while the theoretical data show a slowly vanishing tail the experimental data display a sudden decrease. Our calculations, therefore, imply that magnetic and superconducting order are competing ground states in the sense that magnetism does not die before the onset of superconductivity. The sharp decrease in moment at $x=0.05$ may then be brought about by the onset of superconductivity. The question of whether magnetism and superconductivity then coexist for a small doping range, or if the onset of superconductivity destroys entirely the magnetic order, cannot be answered without explicitly treating superconductivity within the same framework. At this point one should also note that the small moment itinerant magnetism of this system implies a strong role for spin fluctuations $\mathrm{s}^{4,36,37}$ which is not correctly treated by the (semi)local functionals used in the present calculations. These fluctuations may also act to damp the slowly vanishing tail of the moment vs doping seen in Fig. 5.

Similar calculations for $\mathrm{CeO}_{1-x} \mathrm{~F}_{x} \mathrm{FeAs}$ (left panel Fig. 6) show that there is almost no change in the $\mathrm{Fe}$ moment upon doping. This behavior is totally different from that of $\mathrm{LaO}_{1-x} \mathrm{~F}_{x} \mathrm{FeAs}$ and is in apparent contradiction to the experimental data. The reason for this again lies in the magnetic coupling between the $\mathrm{Ce}$ and $\mathrm{Fe}$ atoms; localized $\mathrm{Ce}$ moments are not affected by small doping in the FeAs plane and 


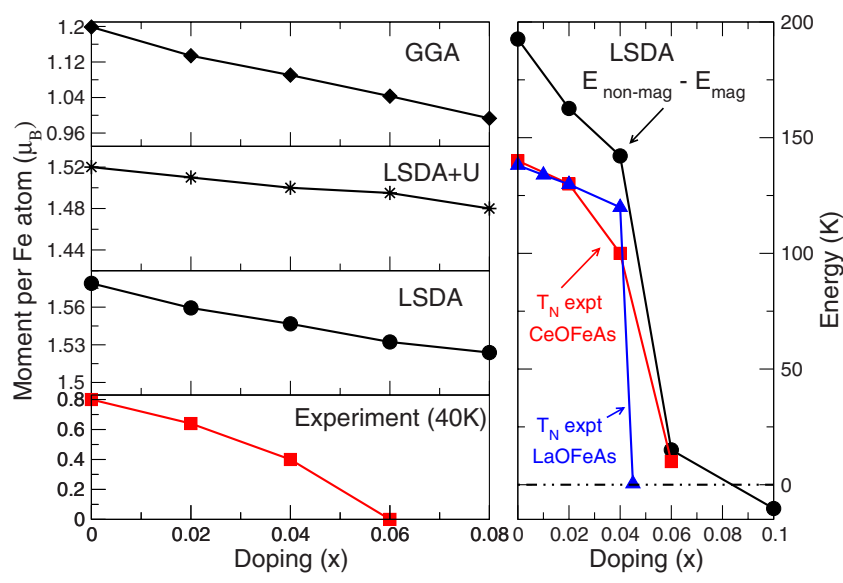

FIG. 6. (Color online) Left panel: moment per Fe atom (in $\mu_{B}$ ) as a function of doping calculated using the LSDA, LSDA $+U$, and GGA. The right panel: shows the difference between the magnetic and the nonmagnetic total energy (in K) calculated using the LSDA. The experimental data for $\mathrm{CeO}_{1-x} \mathrm{~F}_{x} \mathrm{FeAs}$ are taken from Ref. 14 and for $\mathrm{LaO}_{1-x} \mathrm{~F}_{x} \mathrm{FeAs}$ from Ref. 12

reasonably strong coupling between $\mathrm{Ce}$ and $\mathrm{Fe}$ moments then leads to very small change in Fe moments as a function of doping. However, if we consider the magnetization energy, i.e., the quantity $E_{\text {nonmag }}-E_{\text {mag }}$ (right panel Fig. 6), this falls sharply at exactly the critical doping $x_{c}=0.06$. Thus it is the stability of the magnetic order which is reduced upon doping, not the actual Fe moment. This sudden reduction in stability, which is not found in $\mathrm{LaO}_{1-x} \mathrm{~F}_{x} \mathrm{FeAs}$, could be the reason behind the differing forms of the Néel temperature phase boundary in these two compounds.

For the case of LaOFeAs, it is evident from recent experiments ${ }^{21}$ that there exists a complex structural and magnetic behavior with doping, the full nature of which has yet to be clarified. In fact, on the question of whether the distortion observed at $x=0$ persists beyond the superconducting transition at $x=0.05$, the current experimental data are contradictory; Refs. 21 and 32 find that distortion persists up to $x=0.08$, while in Refs. 10 and 12 no distortion is observed beyond the onset of superconductivity at $x=0.05$. The magnetic state of $\mathrm{LaO}_{1-x} \mathrm{~F}_{x} \mathrm{FeAs}$ is also uncertain, with one experiment ${ }^{21}$ finding evidence of an incommensurate structure for $x>0$. In order to clarify this situation we have determined the ground state for several doping concentrations, by minimizing over both the distortion angle $\gamma$ and spiral vector q. Our major findings are (see Fig. 7); (a) up to a doping of $x=0.08$ distorted crystal, with distortion angle $\gamma$ $=90.2^{\circ}$, is lowest in energy. ${ }^{38}$ (b) Beyond the doping of $x$ $=0.08$ the system again prefers to be in a tetragonal ground state (with $\gamma=90^{\circ}$ ). These results are in agreement with the experimental findings. ${ }^{11,21}$ (c) Subsequent minimization of the spin spiral $\mathbf{q}$ reveals a stripe phase to incommensurate spin-spiral transition $(\mathbf{q}=[0.54,0.54,0])$ at a doping of $x$ $=0.04$. This finding is in agreement with the experimental evidence of Huang et al. ${ }^{21}$ We should note, however, that the small energy difference between the incommensurate and

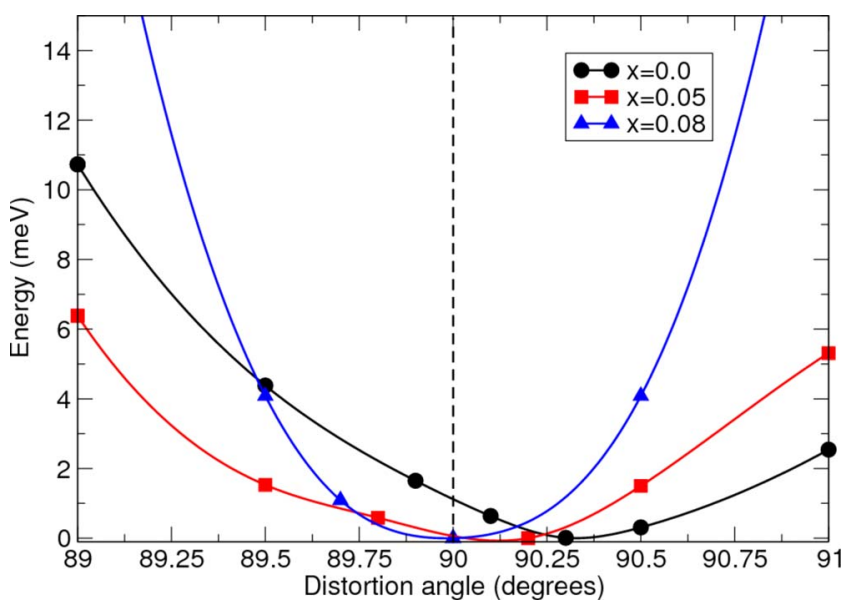

FIG. 7. (Color online) Total energy (in meV) per formula unit as a function of the distortion angle (in degrees). Results are for undoped, $5 \%$ and $8 \%$ doped $\mathrm{LaOFeAs}$, and are calculated using the vBH parameterization for LSDA.

stripe structures $(0.4 \mathrm{meV}$ per formula unit) suggests that this may only be seen at the low-temperature experiments.

On investigating the possible appearance of the incommensurate phase in the doping phase diagram of $\mathrm{CeO}_{1-x} \mathrm{~F}_{x} \mathrm{FeAs}$ we find that in contrast to the case of $\mathrm{LaO}_{1-x} \mathrm{~F}_{x} \mathrm{FeAs}$ (i) the distortion angle is unchanged upon doping $(0 \leq x \leq 0.06)$ and (ii) the ground-state magnetic structure remains stripe AFM.

\section{CONCLUSIONS}

To conclude, we have performed a comparative study of the two oxypnictides $\mathrm{CeO}_{1-x} \mathrm{~F}_{x} \mathrm{FeAs}$ and $\mathrm{LaO}_{1-x} \mathrm{~F}_{x} \mathrm{FeAs}$. Although many common features, e.g., a similar nonmagnetic Fermi surface, may be found in the iron oxypnictides we have shown here that profound differences also exist. In particular we find that the structural distortion in CeOFeAs is driven by one-electron kinetic energy (i.e., band-structure effect), in contrast to LaOFeAs where a gain in magnetization energy has been identified as the mechanism. Furthermore, the behavior under electron doping differs markedly between the two materials; in $\mathrm{CeO}_{1-x} \mathrm{~F}_{x} \mathrm{FeAs}$ we find that while the ground-state moment is essentially unchanged with doping, the stability of the moment is sharply reduced. Finally, we have-via calculations of incommensurate spin-spiral structures - carefully investigated the impact of doping on the crystal structure of $\mathrm{LaOFeAs}$ and CeOFeAs; we find that two materials behave differently in that $\mathrm{CeO}_{1-x} \mathrm{~F}_{x} \mathrm{FeAs}$ stays commensurate with distortion angle $\gamma=90.3^{\circ}$ up to a doping concentration of $6 \%$. While, $\mathrm{LaO}_{1-x} \mathrm{~F}_{x} \mathrm{FeAs}$ becomes incommensurate at a doping of $4 \%$ and further show a transition from orthorhombic to tetragonal structure at a doping of $8 \%$. Given a huge debate about the dependence of the results for oxypnictides on the exchange-correlation functional used, in the present work the calculations are performed using LSDA, GGA, and LSDA $+U$ functionals and it is shown that the above-mentioned conclusions are independent of the choice of the functional. 
*sangeeta.sharma@physik.fu-berlin.de

${ }^{1}$ Y. Kamihara, Takumi Watanabe, Masahiro Hirano, and Hideo Hosono, J. Am. Chem. Soc. 130, 3296 (2008).

${ }^{2}$ O. Millo, I. Asulin, O. Yuli, I. Felner, Z.-A. Ren, X. L. Shen, G. C. Che, and Z. X. Zhao, Phys. Rev. B 78, 092505 (2008).

${ }^{3}$ I. I. Mazin, D. J. Singh, M. D. Johannes, and M. H. Du, Phys. Rev. Lett. 101, 057003 (2008).

${ }^{4}$ I. I. Mazin and M. D. Johannes, Nat. Phys. 5, 141 (2009).

${ }^{5}$ Z. P. Yin, S. Lebegue, M. J. Han, B. P. Neal, S. Y. Savrasov, and W. E. Pickett, Phys. Rev. Lett. 101, 047001 (2008).

${ }^{6}$ L. Pourovskii, V. Vildosola, S. Biermann1, and A. Georges, EPL 84, 37006 (2008)

${ }^{7}$ I. I. Mazin, M. D. Johannes, L. Boeri, K. Koepernik, and D. J. Singh, Phys. Rev. B 78, 085104 (2008).

${ }^{8}$ S. Sharma, J. Dewhurst, S. Shallcross, C. Bersier, F. Cricchio, A. Sanna, S. Massidda, E. Gross, and L. Nordstrom, arXiv:0810.4278 (unpublished).

${ }^{9}$ T. Nomura, S. W. Kim, Y. Kamihara, M. Hirano, P. V. Sushko, K. Kato, M. Takata, A. L. Shluger, and H. Hosono, Supercond. Sci. Technol. 21, 125028 (2008).

${ }^{10}$ H.-H. Klauss, H. Luetkens, R. Klingeler, C. Hess, F. J. Litterst, M. Kraken, M. M. Korshunov, I. Eremin, S.-L. Drechsler, R. Khasanov, A. Amato, J. Hamann-Borrero, N. Leps, A. Kondrat, G. Behr, J. Werner, and B. Büchner, Phys. Rev. Lett. 101, 077005 (2008).

${ }^{11}$ C. de la Cruz, Q. Huang, J. W. Lynn, Jiying Li, W. Ratcliff II, J. L. Zarestky, H. A. Mook, G. F. Chen, J. L. Luo, N. L. Wang, and Pengcheng Dai, Nature (London) 453, 899 (2008).

${ }^{12}$ H. Luetkens H.-H. Klauss, M. Kraken, F. J. Litterst, T. Dellmann, R. Klingeler, C. Hess, R. Khasanov, A. Amato, C. Baines, M. Kosmala, O. J. Schumann, M. Braden, J. Hamann-Borrero, N. Leps, A. Kondrat, G. Behr, J. Werner, and B. Büchner, Nature Mater. 8, 305 (2009).

${ }^{13}$ H.-H. Wen, X. H. Chen, W. L. Yang, and Z. X. Zhao, Phys. Rev. Lett. 85, 2805 (2000).

${ }^{14}$ J. Zhao, Q. Huang, Clarina de la Cruz, Shiliang Li, J. W. Lynn, Y. Chen, M. A. Green, G. F. Chen, G. Li, Z. Li, J. L. Luo, N. L. Wang, and Pengcheng Dai, Nature Mater. 7, 953 (2008).

${ }^{15}$ G. F. Chen, Z. Li, D. Wu, G. Li, W. Z. Hu, J. Dong, P. Zheng, J. L. Luo, and N. L. Wang, Phys. Rev. Lett. 100, 247002 (2008).

${ }^{16}$ D. A. Zocco, J. J. Hamlina, R. E. Baumbacha, M. B. Maplea, M. A. McGuireb, A. S. Sefatb, B. C. Salesb, R. Jinb, D. Mandrusb, J. R. Jeffriesc, S. T. Weirc, and Y. K. Vohra, Physica C 468, 2229 (2008).

${ }^{17}$ T. Yildirim, Phys. Rev. Lett. 101, 057010 (2008).

${ }^{18}$ F. Ma, Z. Y. Lu, and T. Xiang, Phys. Rev. B 78, 224517 (2008).

${ }^{19}$ D. J. Singh and L. Nordström, Planewaves, Pseudopotentials, and the LAPW Method (Springer, New York, 2006).
${ }^{20}$ URL http://elk.sourceforge.net

${ }^{21}$ Q. Huang, J. Zhao, J. W. Lynn, G. F. Chen, J. L. Luo, N. L. Wang, and P. Dai, Phys. Rev. B 78, 054529 (2008).

${ }^{22}$ H. M. Alyahyaei and R. A. Jishi, Phys. Rev. B 79, 064516 (2009).

${ }^{23}$ J. P. Perdew, K. Burke, and M. Ernzerhof, Phys. Rev. Lett. 77, 3865 (1996).

${ }^{24}$ J. P. Perdew and Y. Wang, Phys. Rev. B 45, 13244 (1992).

${ }^{25}$ A. N. Yaresko, G. Q. Liu, V. N. Antonov, and O. K. Andersen, Phys. Rev. B 79, 144421 (2009).

${ }^{26}$ J. Lorenzana, G. Seibold, C. Ortix, and M. Grilli, Phys. Rev. Lett. 101, 186402 (2008).

${ }^{27}$ M. D. Johannes and I. I. Mazin, arXiv:0904.3857 (unpublished).

${ }^{28}$ J. Dong, H. J. Zhang, G. Xu, Z. Li, G. Li, W. Z. Hu, D. Wu, G. F. Chen, X. Dai, J. L. Luo, Z. Fang, and N. L. Wang, EPL 83, 27006 (2008).

${ }^{29}$ M. A. McGuire, Andrew D. Christianson, Athena S. Sefat, Brian C. Sales, Mark D. Lumsden, Rongying Jin, E. Andrew Payzant, David Mandrus, Yanbing Luan, Veerle Keppens, Vijayalaksmi Varadarajan, Joseph W. Brill, Raphaël P. Hermann, Moulay T. Sougrati, Fernande Grandjean, and Gary J. Long, Phys. Rev. B 78, 094517 (2008).

${ }^{30}$ F. Bondino, E. Magnano, M. Malvestuto, F. Parmigiani, M. A. McGuire, A. S. Sefat, B. C. Sales, R. Jin, D. Mandrus, E. W. Plummer, D. J. Singh, and N. Mannella, Phys. Rev. Lett. 101, 267001 (2008)

${ }^{31}$ S. Chi, D. T. Adroja, T. Guidi, R. Bewley, Shiliang Li, Jun Zhao, J. W. Lynn, C. M. Brown, Y. Qiu, G. F. Chen, J. L. Lou, N. L. Wang, and Pengcheng Dai, Phys. Rev. Lett. 101, 217002 (2008).

${ }^{32}$ D. R. Garcia, C. Jozwiak, C. G. Hwang, A. Fedorov, S. M. Hanrahan, S. D. Wilson, C. R. Rotundu, B. K. Freelon, R. J. Birgeneau, E. Bourret-Courchesne, and A. Lanzara, Phys. Rev. B 78, 245119 (2008).

${ }^{33}$ G. Giovannetti, S. Kumar, and J. van den Brink, Physica B 403, 3653 (2008).

${ }^{34}$ P. Larson and S. Satpathy, Phys. Rev. B 79, 054502 (2009).

${ }^{35}$ U. von Barth and L. Hedin, J. Phys. C 5, 1629 (1972).

${ }^{36}$ I. Opahle, H. C. Kandpal, Y. Zhang, C. Gros, and R. Valenti, Phys. Rev. B 79, 024509 (2009).

${ }^{37}$ Y. Kohama, Y. Kamihara, M. Hirano, H. Kawaji, T. Atake, and H. Hosono, Phys. Rev. B 78, 020512(R) (2008).

${ }^{38}$ For these calculations corresponding experimental lattice parameters from Refs. 11 and 21 are used. It is worth noting that on the use of undoped experimental lattice parameter, for the theoretical calculations of the doped $\mathrm{LaOFeAs}$, an erroneous distortion in the opposite direction $(\gamma<90)$ is obtained. 